\title{
Heuristic for Flow Shop Sequencing with Separated and Sequence Independent Setup Times
}

This paper deals with the permutation flow shop scheduling problem with separated and sequence-independent machine setup times. A heuristic method with the objective of minimizing the total time to complete the schedule is introduced. The proposed heuristic is based on a structural property of this scheduling problem, which provides an upper bound on the idle time of the machines between the completion of the setup task and the beginning of job processing. Experimental results show that the new heuristic outperforms two existing ones.

Keywords: production scheduling, permutation flow shop, setup times, heuristic method.

drnagano@usp.br

University of São Paulo

Production Engineering

13566-590 São Carlos, São Paulo, Brazil environment is also common in the printing, textile and plastic industries (Das, Gupta and Khumawala, 1995).

Basically, there are two types of machine setup times which can be dealt with separately from the job processing times. For the first one, the setup time depends only on the operation to be performed, regardless of job sequences, which is sequence-independent. The second type depends on both the operation to be performed and the operation that was processed immediately before on the same machine, and therefore it is sequence-dependent.

This paper deals with the permutation flow shop problem where the operation setup times are separate from the processing times, and are sequence-independent, which will be denoted by the PFS-SIS problem. A new heuristic based on a structural property of this scheduling problem has been introduced by Moccellin and Nagano (2007).

\section{Literature Review}

Analysis of the literature shows that the first work for the PFSSIS problem was developed by Yoshida and Hitomi (1979) for a flow shop with only two machines in order to minimize the makespan. The authors showed that the problem can be optimally solved by using the traditional Johnson's rule (Johnson, 1954).

A number of papers with the objective of finding the optimal solution with minimum makespan emerged later for the same case of two machines, but with additional constraints such as job due dates (Khurana and Bagga, 1985), job removal times, and no-wait job processing (Gupta, Strusevich and Zwaneveld, 1997).

There is other work concerning the optimal solution, however, using performance measures such as minimizing the mean flowtime (Bagga and Khurana, 1986), and minimizing the maximum lateness (Allahverdi and Aldowaisan, 1998).

For flow shops with multiple machines, Yoshida and Hitomi (1979) showed that even for only three machines, the makespan minimization is not necessarily given by a permutation schedule. Even so, for the multiple machine case, the research reported in the literature considers only permutation solutions obtained by heuristic methods. This research attempts to minimize the makespan under constraints such as limited buffers (Park and Steudel, 1991), job transfer time between machines (Cao and Bedworth, 1992) and non-zero job removal time from the machines (Rajendran and Ziegler, 1997).

\section{A Structural Property of the PFS-SIS Problem}

As aforementioned, Moccellin and Nagano (2007) introduced a structural property of the PFS-SIS problem as a result of an investigation into the problem characteristics. This property 
provides an upper bound on the idle time of the machines between the setup task and the job processing, as follows.

Consider the PFS-SIS problem with $m$ machines and $n$ jobs. Let $l u$ and $l_{v}$ be two successive arbitrary jobs, $S_{w v}$ the setup task on machine $M_{k \leftarrow+1}$ for the processing of job $J_{v}$, and $X_{S_{u v^{2}} k+1}^{k}$ the idle time of machine $M_{h+1}$ between the completion of the setup $S_{N v}$ and the

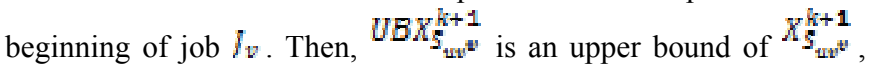
given by:

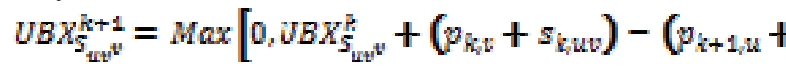$$
\text { For } k=1,2, \ldots ., m-1 \text { with } U B X_{\kappa_{u v^{v}}}^{1}=\mathbf{0} \text {, }
$$

Where

$p_{q j}=$ processing time of job $l j$ on machine $M_{q}$;

$s_{q, u v}=$ setup time of machine $M_{q}$ for processing job $I_{v}$, given that job $l_{\mathrm{h}}$ immediately precedes $l_{v}$.

Expression (1) recursively allows the calculation of the upper bound $U B X_{S \Psi}^{E+1}$

time intervals $X_{S_{u w^{4}}}^{1}$ are zero, that is $U E X_{S_{u w^{4}}}^{1}=\mathbf{0}$. Therefore, by using expression (1) we can calculate the upper bounds of the idle times in the last machine $M_{m}$ regarding the $n(n-1)$ possible arrangements for any job pair from the set of $n$ jobs.

Let $U B X^{m}$ be the $n \times n$ matrix as follows:

$$
U B X^{m}=\left(\begin{array}{ccc}
\infty & \cdots & v B X_{v_{2 n}^{n n}}^{n} \\
\vdots & \cdots & \vdots \\
U B X_{\xi_{11}}^{m} & \cdots & \infty
\end{array}\right)_{i \mathbf{x}_{j}}
$$

Where:

$v B X_{5}^{n}$

${ } w^{j}$ is the upper bound of the idle time in the last machine

$M_{m}$ between the completion of the setup $S_{i j}$ and the beginning of job $l_{j}$, for $i=1,2, \ldots, n$, and $j=1,2, \ldots, n, i \neq j$.

\section{The New Heuristic}

As we have mentioned, the heuristic method we propose (denoted by $\mathrm{MN}$ heuristic) is based on the structural property of the PFS-SIS problem presented in section 3.

The MN heuristic can be stated as follows:

Step 1: Find jobs $J_{x}$ and $J_{y}$ such that

$$
U B K_{S_{s y^{N}}}^{\mathrm{m}}=\min _{\substack{i, j=1, z_{i}-4 \\ i \neq j}} U B X_{S_{H}}^{\mathrm{m}}
$$

Step 2: Set $U B X_{S_{x}^{X}}^{m}=U B X_{S_{y}}^{m}=\infty$

Step 3: Find job $l_{w}$ such that $U B X_{S_{W^{w}}}^{m}=\min _{j=1 / 2, n} U B X_{S_{y}}^{m}$.

Step 4: Set $U B X_{s_{1 w^{w}}^{m}}^{m}=\infty$, for all $i=1,2, \ldots, n$.

Step 5: Set $k=3$, and let $\sigma$ be the $k$-job partial sequence given by $\sigma=\left(l_{x}, l_{y}, h_{w}\right)$.

Step 6: Find the best sequence from the entire Interchange Neighborhood of sequence $\sigma$. If the makespan of the best neighbor is better than that of $\sigma$, assign it to $\sigma$.

Step 7: Find the best sequence from the entire Shift Neighborhood of sequence $\sigma$. If the makespan of the best neighbor is better than that of $\sigma$, assign it to $\sigma$.
If $k=n$, Stop. Sequence $\sigma$ is the MN heuristic solution; otherwise set $k=k+1$, and proceed to Step 8 .

Step 8: Let $h_{L}$ be the job in the last position of sequence $\sigma$.

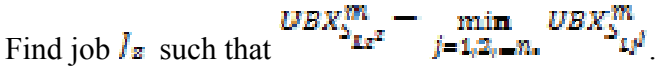
Step 9: Set $U B X_{S_{i z^{x}}^{m}}^{m}=\infty$, for all $i=1,2, \ldots, n$.

Step 10: Let $\sigma^{\prime}$ be a new sequence by adding job $l_{I}$ to the sequence $\sigma$, and placing it in the last position of $\sigma$ '. Assign $\sigma$ ' to $\sigma$, and return to Step 6 .

\section{1,wxisting Heuristics}

The MN heuristic was compared to those proposed by Cao and Bedworth (1992), and Rajendran and Ziegler (1997). Let us denote these heuristics as respectively $\mathrm{CB}$ and RZ heuristics.

As it was mentioned in the section concerning the literature review, Cao and Bedworth (1992) consider the PFS-SIS problem with job transfer times between machines, while Rajendran and Ziegler (1997) deal with non-zero job removal times from the machines. The literature analysis we performed has shown that the PFS-SIS problem without additional constraints has not been considered. Therefore, in order to compare the performance of the MN heuristic with those aforementioned, some simple adaptations have been made, that is, it is enough to make both the job transfer times between machines (Cao and Bedworth, 1992) and the job removal times from the machines equal to zero (Rajendran and Ziegler, 1997). These existing heuristics are described below.

\section{The CB Heuristic (with zero job transfer times)}

The heuristic method by Cao and Bedworth (1992) consists of three phases. In the first phase, an initial solution (job sequence) is obtained by using a procedure similar to the well-known CDS algorithm (Campbell, Dudek and Smith, 1970). The second phase attempts to improve the initial job sequence by a sorting search. Finally, a refinement procedure is used in order to obtain better solutions.

\section{The RZ Heuristics (with zero job removal times)}

Rajendran and Ziegler (1997) have proposed three interrelated heuristics, which will be denoted by RZ1, RZ2, and RZ3 heuristics, as follows:

\section{RZ1 Heuristic}

The RZ1 heuristic consists of two phases. In the first one, an initial solution (job sequence) is obtained which is chosen from a set of $2(m-1)$ generated sequences. Then, the second phase attempts to improve the initial solution by using a job insertion procedure.

\section{RZ2 Heuristic}

The RZ2 heuristic differs from RZ1 only by the Phase 1, i.e., the generation of an initial solution (seed sequence).

\section{RZ3 Heuristic}

The RZ3 heuristic takes the better sequence from the solution sequences yielded by RZ1 and RZ2 heuristics as the seed sequence to be given as input to the improvement procedure concerning Phase 2 of the RZ1 and RZ2 algorithms. Therefore, it can be clearly observed that the solution sequence obtained by the RZ3 heuristic is as good as, or better than the solution sequences yielded by the heuristics RZ1 and RZ2. Therefore, in the comparison experiment the RZ3 heuristic is only considered. 


\section{Computational Experience}

The computational experience was performed on a total of 1,200 problem instances, which comprise two groups called small Flow Shop and large Flow Shop. In the first Flow Shop group, there are a number of machines $m \in\{3,6\}$ and a number of jobs $n \in\{15,20$, $25,30\}$. In the large Flow Shops, $m \in\{10,20\}$ and $n \in\{50,100$, $150,200\}$. The operation processing times were randomly generated by using an integer uniform distribution over the interval $[1,100]$. The machine setup times were randomly generated from three integer uniform distributions over the intervals $[0,50],[0,100]$, and $[0,150]$. Each of the $m \times n \times$ setup time combinations was replicated 25 times. All these problem instances are available at the URL http://soa.iti.es/instancias-de-problemas/. In the computational tests, all the heuristics were coded in Delphi and were run on a microcomputer Pentium $4 \mathrm{CPU} 3.00 \mathrm{GHz}$.

The statistics we have used are the Percentage of Success (in finding the best solution), the Relative Deviation (between the heuristics), and the computation time.
The percentage of success is given by the number of times the heuristic obtains the best makespan (alone or in conjunction with others) divided by the number of solved problems. The relative deviation is defined as

$$
R D_{h}=\frac{\left(n_{h}-n^{*}\right)}{D^{*}}
$$

Where $D_{\boldsymbol{h}}$ is the makespan of the best sequence that was found by heuristic $h$, and $D^{*}$ the best makespan that was found by the heuristics, for a given instance.

The results from the computational experience are shown in Tables 1 to 5 .

Tables 1 to 2 are related to the small Flow Shops, whereas Tables 3 to 5 present results concerning the large ones.

Table 1. Percentage of Success for Small Flow Shops.

\begin{tabular}{|c|c|c|c|c|c|c|c|c|c|c|}
\hline \multirow[b]{2}{*}{$n$} & \multirow[b]{2}{*}{$m$} & \multicolumn{3}{|c|}{ setup $[0,50]$} & \multicolumn{3}{|c|}{ setup $[0,100]$} & \multicolumn{3}{|c|}{ setup $[0,150]$} \\
\hline & & CB & RZ3 & MN & CB & RZ3 & MN & CB & RZ3 & $\mathbf{M N}$ \\
\hline 15 & 3 & 0.48 & 0.6 & 0.72 & 0.28 & 0.4 & 0.96 & 0.32 & 0.4 & 0.92 \\
\hline 15 & 6 & 0.08 & 0.16 & 0.84 & 0.08 & 0.04 & 0.92 & 0.04 & 0.04 & 0.92 \\
\hline 20 & 3 & 0.48 & 0.6 & 0.92 & 0.36 & 0.52 & 0.96 & 0.44 & 0.36 & 0.96 \\
\hline 20 & 6 & 0.12 & 0 & 0.92 & 0.04 & 0.08 & 0.96 & 0.08 & 0.04 & 0.92 \\
\hline 25 & 3 & 0.32 & 0.4 & 0.84 & 0.52 & 0.52 & 0.96 & 0.36 & 0.48 & 0.96 \\
\hline 25 & 6 & 0.08 & 0 & 0.96 & 0.04 & 0.08 & 0.92 & 0 & 0.04 & 0.96 \\
\hline 30 & 3 & 0.36 & 0.56 & 1 & 0.32 & 0.56 & 0.96 & 0.44 & 0.48 & 0.96 \\
\hline 30 & 6 & 0 & 0.08 & 0.92 & 0.04 & 0 & 0.96 & 0.12 & 0 & 0.96 \\
\hline \multicolumn{2}{|c|}{ Average } & 0.24 & 0.3 & 0.89 & 0.21 & 0.24 & 0.95 & 0.23 & 0.23 & 0.95 \\
\hline
\end{tabular}

Table 1 shows that the MN heuristic performance is much better than the others. The average percentage of success regarding the proposed heuristic is $93 \%$ in comparison with $26 \%$ for the RZ3 heuristic, and $23 \%$ for the $\mathrm{CB}$ one. Moreover, the MN performance is better for greater setup times.
It is interesting to note that $\mathrm{CB}$ and $\mathrm{RZ} 3$ performances decrease as the number of machines increases. However, this does not occur for the the MN heuristic.

Table 2. Relative Deviation for Small Flow Shops.

\begin{tabular}{|c|c|c|c|c|c|c|c|c|c|c|}
\hline \multirow{3}{*}{$n$} & \multirow{3}{*}{$m$} & \multirow{2}{*}{\multicolumn{3}{|c|}{ setup $[0,50]$}} & \multirow{2}{*}{\multicolumn{3}{|c|}{ setup $[0,100]$}} & \multirow{2}{*}{\multicolumn{3}{|c|}{ setup $[0,150]$}} \\
\hline & & & & & & & & & & \\
\hline & & CB & RZ3 & MN & CB & RZ3 & MN & CB & RZ3 & MN \\
\hline 15 & 3 & 0.81 & 0.6 & 0.16 & 1.24 & 0.62 & 0 & 1.12 & 1.14 & 0.08 \\
\hline 15 & 6 & 2.68 & 2.69 & 0.29 & 2.7 & 3.51 & 0.03 & 3.08 & 4.05 & 0.17 \\
\hline 20 & 3 & 0.76 & 0.34 & 0.03 & 0.93 & 0.64 & 0.01 & 0.97 & 0.95 & 0 \\
\hline 20 & 6 & 2.09 & 3.24 & 0.05 & 3.13 & 3.45 & 0.06 & 3.51 & 4.49 & 0.09 \\
\hline 25 & 3 & 1.13 & 0.52 & 0.1 & 0.89 & 0.94 & 0 & 0.62 & 0.35 & 0.01 \\
\hline 25 & 6 & 2.72 & 3.14 & 0.01 & 3.43 & 3.05 & 0.02 & 3.38 & 2.8 & 0.02 \\
\hline 30 & 3 & 0.9 & 0.5 & 0 & 0.8 & 0.48 & 0.02 & 0.46 & 0.39 & 0 \\
\hline 30 & 6 & 2.96 & 2.87 & 0.06 & 2.45 & 2.11 & 0.02 & 2.46 & 2.77 & 0 \\
\hline \multicolumn{2}{|c|}{ Average } & 1.75 & 1,73 & 0.08 & 1.94 & 1.85 & 0.02 & 1.95 & 2.11 & 0.04 \\
\hline
\end{tabular}


The relative deviations from Table 2 confirm the results related to the percentages of success. Moreover, the MN heuristic has a better performance for instances in which the setup times are generated in the interval $[0,100]$ because the average relative deviation is the smallest $(0.02 \%)$. It is also noted that $\mathrm{CB}$ and RZ3 heuristics have better performances for small setup times (interval [0, 50]).

For small Flow Shops the computation times were negligible. The problem instances with 6 machines and 30 jobs have been solved spending 0.05 seconds.
The results from Table 3 related to large Flow Shops are surprising, as the MN heuristic obtained $100 \%$ of success in finding the best solution.

The results from Tables 3 and 4 also show that CB and RZ3 performances decrease as the number of machines increases. It can be noted that for large Flow Shops, the MN performance tends to be independent in the setup time ranges.

Table 3. Percentage of Success for Large Flow Shops.

\begin{tabular}{|c|c|c|c|c|c|c|c|c|c|c|}
\hline \multirow[b]{2}{*}{$n$} & \multirow[b]{2}{*}{$m$} & \multicolumn{3}{|c|}{ setup [0, 50] } & \multicolumn{3}{|c|}{ setup $[0,100]$} & \multicolumn{3}{|c|}{ setup $[0,150]$} \\
\hline & & $\mathbf{C B}$ & RZ3 & $\mathbf{M N}$ & $\mathbf{C B}$ & RZ3 & $\mathbf{M N}$ & CB & RZ3 & MN \\
\hline 50 & 10 & 0.00 & 0.00 & 1.00 & 0.00 & 0.00 & 1.00 & 0.00 & 0.00 & 1.00 \\
\hline 50 & 20 & 0.00 & 0.00 & 1.00 & 0.00 & 0.00 & 1.00 & 0.00 & 0.00 & 1.00 \\
\hline 100 & 10 & 0.00 & 0.00 & 1.00 & 0.00 & 0.00 & 1.00 & 0.00 & 0.00 & 1.00 \\
\hline 100 & 20 & 0.00 & 0.00 & 1.00 & 0.00 & 0.00 & 1.00 & 0.00 & 0.00 & 1.00 \\
\hline 150 & 10 & 0.00 & 0.00 & 1.00 & 0.00 & 0.00 & 1.00 & 0.00 & 0.00 & 1.00 \\
\hline 150 & 20 & 0.00 & 0.00 & 1.00 & 0.00 & 0.00 & 1.00 & 0.00 & 0.00 & 1.00 \\
\hline 200 & 10 & 0.00 & 0.00 & 1.00 & 0.00 & 0.00 & 1.00 & 0.00 & 0.00 & 1.00 \\
\hline 200 & 20 & 0.00 & 0.00 & 1.00 & 0.00 & 0.00 & 1.00 & 0.00 & 0.00 & 1.00 \\
\hline
\end{tabular}

Table 4. Relative Deviation for Large Flow Shops.

\begin{tabular}{|c|c|c|c|c|c|c|c|c|c|c|}
\hline \multirow[b]{2}{*}{$\boldsymbol{n}$} & \multirow[b]{2}{*}{$m$} & \multicolumn{3}{|c|}{ setup [0, 50] } & \multicolumn{3}{|c|}{ setup $[0,100]$} & \multicolumn{3}{|c|}{ setup $[0,150]$} \\
\hline & & CB & RZ3 & $\mathbf{M N}$ & CB & RZ3 & $\mathbf{M N}$ & CB & RZ3 & MN \\
\hline 50 & 10 & 4.12 & 4.25 & 0.00 & 4.62 & 4.43 & 0.00 & 4.68 & 5.41 & 0.00 \\
\hline 50 & 20 & 5.58 & 5.01 & 0.00 & 6.11 & 5.76 & 0.00 & 5.91 & 6.28 & 0.00 \\
\hline 100 & 10 & 4.80 & 4.28 & 0.00 & 4.40 & 4.01 & 0.00 & 4.83 & 4.45 & 0.00 \\
\hline 100 & 20 & 7.06 & 5.46 & 0.00 & 6.85 & 5.86 & 0.00 & 6.76 & 6.07 & 0.00 \\
\hline 150 & 10 & 4.45 & 3.82 & 0.00 & 4.19 & 3.29 & 0.00 & 3.94 & 3.60 & 0.00 \\
\hline 150 & 20 & 6.65 & 5.46 & 0.00 & 6.54 & 5.52 & 0.00 & 7.23 & 5.75 & 0.00 \\
\hline 200 & 10 & 4.56 & 3.24 & 0.00 & 4.36 & 3.33 & 0.00 & 4.36 & 3.60 & 0.00 \\
\hline 200 & 20 & 7.07 & 4.94 & 0.00 & 6.96 & 5.29 & 0.00 & 6.91 & 5.64 & 0.00 \\
\hline \multicolumn{2}{|c|}{ Average } & 5.53 & 4.55 & 0.00 & 5.50 & 4.68 & 0.00 & 5.57 & 5.10 & 0.00 \\
\hline
\end{tabular}

Table 5. Computation Time for Large Flow Shops.

\begin{tabular}{cccccccccccc} 
& & \multicolumn{3}{c}{ setup [0, 50] } & \multicolumn{3}{c}{ setup [0, 100] } & \multicolumn{3}{c}{ setup [0, 150] } \\
\hline $\boldsymbol{n}$ & $\boldsymbol{m}$ & $\mathbf{C B}$ & $\mathbf{R Z 3}$ & $\mathbf{M N}$ & $\mathbf{C B}$ & $\mathbf{R Z 3}$ & MN & \multicolumn{2}{c}{ CB } & RZ3 & MN \\
\hline $\mathbf{5 0}$ & $\mathbf{1 0}$ & 0.02 & 0.09 & 0.57 & 0.02 & 0.08 & 0.57 & 0.04 & 0.08 & 0.57 \\
$\mathbf{5 0}$ & $\mathbf{2 0}$ & 0.07 & 0.17 & 1.16 & 0.08 & 0.17 & 1.17 & 0.08 & 0.17 & 1.18 \\
$\mathbf{1 0 0}$ & $\mathbf{1 0}$ & 0.11 & 0.63 & 8.53 & 0.11 & 0.62 & 8.34 & 0.11 & 0.61 & 8.31 \\
$\mathbf{1 0 0}$ & $\mathbf{2 0}$ & 0.36 & 1.33 & 18.36 & 0.36 & 1.33 & 17.68 & 0.36 & 1.32 & 17.62 \\
$\mathbf{1 5 0}$ & $\mathbf{1 0}$ & 0.31 & 2.09 & 44.29 & 0.31 & 2.09 & 41.33 & 0.32 & 2.07 & 41.31 \\
$\mathbf{1 5 0}$ & $\mathbf{2 0}$ & 0.93 & 4.48 & 89.07 & 0.93 & 4.46 & 87.35 & 0.94 & 4.45 & 88.54 \\
$\mathbf{2 0 0}$ & $\mathbf{1 0}$ & 0.66 & 5.04 & 129.91 & 0.66 & 5.01 & 129.73 & 0.65 & 4.98 & 129.42 \\
$\mathbf{2 0 0}$ & $\mathbf{2 0}$ & 1.88 & 10.56 & 274.15 & 1.88 & 10.52 & 272.73 & 1.89 & 10.54 & 275.28
\end{tabular}


As can be seen from Table 5, the MN computation time for large Flow Shops is the unique disadvantage in comparison with the others. For the largest instances, having 200 jobs in 20 machines, the mean CPU time was equal to 274.05 seconds. However, the trade-off between solution quality and computational effort is a favorable feature of the proposed heuristic.

\section{Final Remarks}

In this paper, a new heuristic method for the permutation flow shop scheduling problem with separated and sequence-independent machine setup times was introduced, with the objective of minimizing the makespan. This heuristic is based on a structural property of this scheduling problem, which provides an upper bound on the idle time of the machines between the setup task and job processing. A computational experience was carried out in order to evaluate the performance of the proposed heuristic in comparison with heuristics reported in the literature. The experimental results showed that the new heuristic is clearly superior to the existing ones, even though more computational effort was required. Therefore, in order to compare the performance of the new heuristic, some simple adaptations have been made, that is, it is enough to make both the job transfer times between machines and the job removal times from the machines equal to zero. One possible extension of our work is to find some heuristics without additional adaptations. Another possible extension is to address the problem with respect to mean tardiness or mean flowtime.

\section{Acknowledgements}

The authors wish to thank the anonymous referees for their constructive comments and suggestions on earlier versions of this paper. The research reported in this paper was partially supported by a grant from the National Council for Scientific and Technological Development (CNPq), Brazil.

\section{References}

Allahverdi, A., Aldowaisan, T., 1998, "Job lateness in flowshops with setup and removal times separated", Journal of the Operational Research Society, Vol. 49, pp. 1001-1006.
Bagga, P.C., Khurana, K., 1986, "Two-machine flowshop with separated sequence-independent setup times: mean completion time criterion", Indian Journal of Management and Systems, Vol. 2, pp. 47-57.

Cao, J., Bedworth, D.C., 1992, "Flow shop scheduling in serial multiproduct processes with transfer and setup times", International Journal of Production Research, Vol. 30, pp. 1819-1830.

Campbell, H., Dudek, R., Smith, M., 1970, "A heuristic algorithm for the n-job m-machine sequencing problem", Management Science, Vol. 16B, pp. 630-637.

Cheng, T.C.E., Gupta, J.N.D., Wang, G., 2000, “A review of flowshop scheduling research with setup times", Production and Operations Management, Vol. 9, pp. 262-282.

Das, S.R., Gupta, J.N.D., Khumawala, B.M., 1995, "A savings index heuristic algorithm for flowshop scheduling with sequence dependent set-up times", Journal of the Operational Research Society, Vol. 46, pp. 1365-1373.

Gupta, J.N.D., Stafford Jr., E.F., 2006, "Flowshop scheduling research after five decades", European Journal of Operational Research, Vol. 169, pp. 699-711.

Gupta, J.N.D., Strusevich, V.A., Zwaneveld, C., 1997. "Two-stage nowait scheduling models with setup and removal times", Computers and Operations Research, Vol. 24, pp. 1025-1031.

Hejazi, S.R., Saghafian, S., 2005, "Flowshop-scheduling problems with makespan criterion: a review", International Journal of Production Research, Vol. 43, pp. 2895-2929.

Johnson, S., 1954, "Optimal two- and three-stage production schedules with setup times included", Naval Research Logistics Quarterly, Vol. 1, pp. 61-68.

Khurana, K., Bagga, P.C., 1985, "Scheduling of job-block with deadline in n X 2 flowshop problem with separated setup times", Indian Journal of Pure Applied Mathematics, Vol. 16, pp. 213-224.

Moccellin, J.V., Nagano, M.S., 2007, "Uma propriedade estrutural do problema de programação da produção flow shop permutacional com tempos de setup", Pesquisa Operacional, Vol. 27, pp. 487-515 (in Portuguese).

Park, T., Steudel, H.J., 1991, "Analysis of heuristics for job sequencing in manufacturing flow line work-cells", Computers and Industrial Engineering, Vol. 20, pp. 129-140.

Rajendran, C., Ziegler, H., 1997, "Heuristics for scheduling in a flowshop with setup, processing and removal times separated", Production Planning and Control, Vol. 8, pp. 568-576.

Simons Jr., J.V., 1992, "Heuristics in flow shop scheduling with sequence dependent setup times", Omega, Vol. 20, pp. 215-225.

Yoshida, T., Hitomi, K., 1979, "Optimal two-stage production scheduling with setup times separated", AIEE Transactions, Vol. 11, pp. 261-263. 